\title{
A state-space algorithm for the spectral factorization ${ }^{1}$
}

\author{
F. Kraffer, H. Kwakernaak \\ Systems and Control Group, Department of Applied Mathematics, Twente University, \\ P. O. Box 217, 7500 AE Enschede, The Netherlands. Fax : +31.53.434 0733, \\ e-mails : F.Kraffer@math.utwente.nl, H.Kwakernaak@math.utwente.nl
}

\begin{abstract}
This paper presents an algorithm for the spectral factorization of a para-Hermitian polynomial matrix. The algorithm is based on polynomial matrix to state space and vice versa conversions, and avoids elementary polynomial operations in computations; It relies on well-proven methods of numerical linear algebra such as Schur decompositions. Keywords: Subspace methods, Numerical methods, Linear systems.
\end{abstract}

\section{Introduction}

Polynomial matrices play an important role in linear systems and control theory. The present algorithm for the spectral factorization of a diagonally reduced para-Hermitian polynomial matrix $Z$ is useful in control with quadratic cost functionals. We look for a square polynomial matrix $Q$ and a signature matrix $J$ such that

$$
Z(s)=Q^{*}(s) J Q(s), \quad J=\left[\begin{array}{cc}
I_{m} & 0 \\
0 & -I_{p}
\end{array}\right] \text {. }
$$

The roots of $Q$ all lie in the open left-half plane and the column degrees of $Q$ equal the half-diagonaldegrees of $Z$. Sufficient but not necessary for the existence of such a factorization is that $Z$ has no roots on the imaginary axis. If the factorization (1) exists such that $Q$ has the correct column degrees then the factorization is said to be canonical.

A square polynomial matrix $Z$ is para-Hermitian if $Z^{*}=Z$ where the adjoint $Z^{*}$ is the polynomial matrix defined by $Z^{*}(s)=Z^{\mathrm{H}}(-s)$. The $m \times m$ para-Hermitian $Z$ is diagonally reduced if there exist half-diagonal degrees $\delta_{1}, \delta_{2}, \cdots$, $\delta_{m}$, so that the leading diagonal coefficient matrix

$$
Z_{\mathrm{L}}=\lim _{|s| \rightarrow \infty} D^{-1}(-s) Z(s) D^{-1}(s)
$$

exists and is nonsingular. $D$ is the diagonal matrix $D(s)=$ $\operatorname{diag}\left(s^{\delta_{1}}, s^{\delta_{2}}, \cdots, s^{\delta_{m}}\right)$.

If $Z$ is not diagonally reduced then it may be made so by a symmetric unimodular transformation [1]. Other algorithms for spectral factorization are described in $[2,3]$. The present algorithm is simpler, and does not require elementary polynomial operations.

\footnotetext{
${ }^{1}$ This paper is a part of the COPERNICUS Project CP 93:2424 in cooperation with the Institute of Information Theory and Automation in Prague, Czech Republic.
}

\section{The algorithm}

The algorithm for (1) is viewed as a special case of $Z=S R$, where $S$ has all its roots in the open right-half plane and $R$ has all its roots in the open left-half plane. Additionally, $R$ is column reduced, the column degrees of $R$ equal the halfdiagonal degrees of $Z$, and $S$ has a special form $S=R^{*} K^{-1}$, with $K$ a constant real nonsingular symmetric matrix. That is, (1) is initially sought in a form

$$
Z(s)=R^{*}(s) K^{-1} R(s) .
$$

1. Find $\frac{d}{d t} x(t)=A x(t), w(t)=C x(t)$ as an observable state-space realization of the differential equation $Z\left(\frac{d}{d t}\right) w(t)=0$.

2. Use Schur transformation to transform the coordinates of the realization $(A, C)$ such that

$$
\begin{aligned}
A & =U^{\mathrm{H}}\left[\begin{array}{cc}
A_{11} & A_{12} \\
0 & A_{22}
\end{array}\right] U, \\
C & =\left[\begin{array}{cc}
C_{1} & C_{2}
\end{array}\right] U,
\end{aligned}
$$

where $A_{11}$ has all its roots in the open left-half plane and $A_{22}$ has all its roots in the open right-half plane.

3. Convert $C_{1}\left(s I-A_{11}\right)^{-1}=R^{-1}(s) E(s)$ such that $R$ is a square polynomial matrix whose column degrees equal those of $Z$.

4. Then $R(s) Z^{-1}(s) R^{*}(s)=K$, where $K$ is a constant Hermitian matrix $K=R(0) Z^{-1}(0) R^{*}(0)$.

5. If $R(s)$ is nonsingular ${ }^{1}$, then $K$ is nonsingular and

$$
Z(s)=R^{*}(s) K^{-1} R(s) \text {. }
$$

The desired spectral factorization $Z(s)=Q^{*}(s) J Q(s)$ with $Q(s)=V R(s)$, follows from the decomposition $K^{-1}=$ $V^{\mathrm{H}} J V$. The constant matrix $V$ is obtained from the Schur decomposition of $L^{-1}$ by permutation.

Separately, if $Z(s)=P^{*}(s) W P(s)$ as in $\mathcal{H}_{\infty}$ applications, then we define $W P(s) w=z$. The system $Z(s) w=0$ is equivalently represented by the two equations $P(s) w=$ $W^{-1} z$ and $P^{*}(s) z=0$, or

$$
\underbrace{\left[\begin{array}{cc}
P(s) & -W^{-1} \\
0 & P^{*}(s)
\end{array}\right]}_{T(s)}\left[\begin{array}{l}
w \\
z
\end{array}\right]=0 .
$$

\footnotetext{
${ }^{1}$ A polynomial matrix is nonsingular if it is square and its determinant is not identically zero.
} 
The realization of (4) is in the form

$$
\frac{d}{d t} x(t)=A x(t), \quad\left[\begin{array}{c}
w(t) \\
z(t)
\end{array}\right]=C x(t) .
$$

Omitting the equation for $z$ results in the desired observable realization of $P^{*}(s) W P(s) w=0$.

\section{The state-space realization}

The state-space realization does not require computation of elementary polynomial operations. The algorithm is a special case of [4]. The polynomial matrix operator $Z(s)$ is considered as a matrix polynomial $Z(s)=Z_{z} s^{z}+\cdots+Z_{1} s+$ $Z_{0}$ in the differential operator $s=\frac{d}{d t}$. The 'external' variables $w: \mathbb{R} \rightarrow \mathbb{R}^{m}$ are from the set of all infinitely often differentiable functions.

1. Introduce 'internal' variables $\xi$ that convert $Z(s) w=0$ to an externally equivalent ${ }^{2}$ form

$$
\begin{array}{r}
P(s) \xi=0, \\
Q \xi \\
{\left[\begin{array}{c}
P(s) \\
Q
\end{array}\right]=\left[\begin{array}{ccccccc}
I & -s I & 0 & \cdots & \ldots & 0 \\
0 & \ddots & \ddots & \ddots & & \vdots \\
\vdots & \ddots & \ddots & \ddots & \ddots & \vdots \\
0 & \cdots & 0 & I & \ddots & 0 \\
Z_{z} & \cdots & \cdots & Z_{1} & Z_{0} & -s I \\
0 & \cdots & \cdots & \cdots & 0 & I \\
\hline 0 & \cdots & \cdots & 0 & I & 0
\end{array}\right]}
\end{array}
$$

Permutation of the 'internal' variables $\xi$ transforms (6) into

$$
\left[\begin{array}{c}
P(s) \\
Q
\end{array}\right]=\left[\begin{array}{cc}
s I-A & -B \\
C & 0 \\
\hline H & 0
\end{array}\right]
$$

2. Compute a state-to-input feedback matrix $F$ such that the maximal $(A, \operatorname{span} B)$-controlled invariant subspace contained in ker $C$ becomes $(A+B F)$-invariant ${ }^{3}$. Use the relevant basis transformation to convert the system description into

$$
\left[\begin{array}{c}
P(s) \\
Q
\end{array}\right]=\left[\begin{array}{ccc}
s I-A_{11} & -A_{12} & -B_{1} \\
0 & s I-A_{22} & -B_{2} \\
0 & C_{2} & 0 \\
\hline H_{1} & H_{2} & 0
\end{array}\right]
$$

where $A_{11}$ determines the maximal controlled invariant subspace. Correspondingly, $\left(A_{22}, B_{2}, C_{2}\right)$ is a strongly observable state-space realization.

3. According to [7], Theorem 1.8, a state-space realization is strongly observable if and only if it has no zeros. Since

$$
U(s):=\left[\begin{array}{cr}
s I-A_{22} & -B_{2} \\
C_{2} & 0
\end{array}\right]
$$

is a square polynomial matrix with no finite zeros, $U(s)$ is unimodular. $U(s)$ guarantees existence of a transformation matrix such that an equivalent system description is in the

\footnotetext{
${ }^{2}$ Operational form for external equivalence is explained in [5].

${ }^{3} \mathrm{~A}$ standard reference for invariant and controlled invariant subspaces in control theory is [6].
}

form

$$
\begin{aligned}
\frac{d}{d t} x(t) & =A_{11} x(t) \\
w(t) & =H_{1} x(t) .
\end{aligned}
$$

where $A_{11}$ and $H_{1}$ are directly inherited from (8). By construction, (10) is externally equivalent to $Z\left(\frac{d}{d t}\right) w(t)=0$.

\section{Conclusions}

Given a diagonally reduced para-Hermitian polynomial matrix $Z$, the result of the algorithm is a square column-reduced polynomial matrix $Q$ with the column degrees equal to the half-diagonal degrees of $Z$ and such that the roots of $Q$ all lie in the open left-half plane; $Z=Q^{*} J Q$ with $J$ the signature.

In case of a nearly noncanonical factorization [2], the algorith stops at $Z(s)=R^{*}(s) K^{-1} R(s)$ with $R$ square columnreduced polynomial matrix with correct column degrees and roots. $K$ is a constant real nearly singular symmetric matrix. The nearly noncanonical form is applicable in $\mathcal{H}_{\infty}$ optimization [3].

The contribution is that the algorithm avoids computation of elementary polynomial operations, and relies on standard numerical linear algebra for constant matrix computations. ${ }^{4}$ The principal application of the algorithm is that of a building block for higher-level algorithms including $\mathcal{H}_{2}$ - and $\mathcal{H}_{\infty}$ optimization [3].

\section{References}

[1] F. M. Callier, "On polynomial matrix spectral factorization by symmetric extraction," IEEE Trans. Aut. Control, vol. AC-30, pp. 453-464, 1985.

[2] Kwakernaak H. and Šebek M., "Polynomial $J$ spectral factorization," IEEE Trans. Aut. Control, vol. AC39, pp. 315-328, Feb. 1994.

[3] H. Kwakernaak, Frequency domain solution of the standard $\mathcal{H}_{\infty}$ problem, pp. 57-107, Springer-Verlag, Berlin, etc, 1996.

[4] F. Kraffer, "Row reduction without polynomial operations, an algorithm," in 4th European Control Conference ECC97, Brussels, Belgium, July 1997.

[5] J. M. Schumacher, "Linear systems under external equivalence," Linear Algebra and its Applications, vol. 102, pp. 1-33, 1988.

[6] Basile, G. and Marro G., Controlled and conditioned invariants in linear system theory, Prentice Hall, Englewood Cliffs, N.J., 1992, incl. Diskette 3.5 inch, ISBN 909006122-3.

[7] M. L. J. Hautus, "Strong Detectability and Observers," Linear Algebra and its Applications, vol. 50, pp. 353-368, 1983.

\footnotetext{
${ }^{4}$ This is important for safeguarding the numerical properties of the algorithm. Current implementation is based on MATLAB kernel and the software appendix to [6].
} 